\section{Bone marrow characteristics predict outcome in a multicenter cohort of primary immune thrombocytopenia patients treated with thrombopoietin analogs}

It is well established that immune thrombocytopenia (ITP) results from increased immune mediated platelet destruction (anti-platelets antibodies, autoreactive $\mathrm{T}$ cells, and reduction of regulatory $\mathrm{T}$ cells) along with impaired production in the bone marrow. ${ }^{1}$ The latter has been attributed to both cellular and humoral mediators that cause suppression of megakaryocyte production and maturation. ${ }^{2}$ Current standard first-line therapy consists of corticosteroids, with or without intravenous Ig, achieving about $70-80 \%$ response rate. However, a consistent proportion of patients would relapse after corticosteroid discontinuation or tapering, and requires further therapy. Second-line treatments encompass the choice among rituximab, splenectomy, and, more recently, the thrombopoietin analogs (TPOa) romiplostim

Table 1. Clinical and bone marrow characteristics of immune thrombocytopenia patients divided according to thrombopoietin analogs treatment.

\begin{tabular}{|c|c|c|c|}
\hline & All cases $N=86$ & Eltrombopag $N=67$ & Romiplostim N=19 \\
\hline \multicolumn{4}{|l|}{ Clinical characteristics } \\
\hline $\mathrm{M}, \mathrm{N}(\%)$ & $35(40.6)$ & $26(38.8)$ & $9(47.4)$ \\
\hline $\mathrm{F}, \mathrm{N}(\%)$ & $51(59.4)$ & $41(61.2)$ & $10(52.6)$ \\
\hline Median age, years (range) & $63(6-87)$ & $63(6-87)$ & $61(20-79)$ \\
\hline Median Plt, x10/L (range) & $17.5(1-99)$ & $21(1-99)$ & $7(1-49)$ \\
\hline *Anti-PLT +, N(\%) & $28(52)$ & $24(51)$ & $4(17.3)$ \\
\hline Bleeding, N(\%) & $50(58.1)$ & $37(55.2)$ & $13(68.4)$ \\
\hline Grade I-II, N & $8-23$ & $5-18$ & $3-5$ \\
\hline Grade III-IV, N & $16-3$ & $12-2$ & $4-1$ \\
\hline \multicolumn{4}{|l|}{ Therapy lines } \\
\hline Steroids, N(CR/PR) & $86(23 / 36)$ & $67(15 / 29)$ & $19(8 / 7)$ \\
\hline Splenectomy, N(CR/PR) & $20(9 / 5)$ & $14(6 / 3)$ & $6(3 / 2)$ \\
\hline Rituximab, N(CR/PR) & $19(2 / 8)$ & $14(1 / 6)$ & $5(1 / 2)$ \\
\hline Cyclosporine, N(CR/PR) & $16(3 / 3)$ & $12(1 / 2)$ & $4(2 / 1)$ \\
\hline Azathioprine, N(CR/PR) & $5(0 / 3)$ & $5(0 / 3)$ & 0 \\
\hline Danazol, N(CR/PR) & $8(1 / 3)$ & $8(1 / 3)$ & 0 \\
\hline \multicolumn{4}{|l|}{ Bone marrow histology } \\
\hline \multicolumn{4}{|l|}{ Cellularity } \\
\hline Median \% cellularity (range) & $40(8-90)$ & $40(8-90)$ & $47(30-80)$ \\
\hline Normal, N(\%) & $39(45.3)$ & $27(40.3)$ & $12(63.1)$ \\
\hline Hyper, N(\%) & $26(30.2)$ & 21(31.3) & $5(26.3)$ \\
\hline Нypo, N(\%) & 18(20.9) & $16(23.9)$ & $2(10.5)$ \\
\hline \multicolumn{4}{|l|}{ Megakaryocytes } \\
\hline Normal, N(\%) & $22(25.6)$ & $15(22.4)$ & $7(36.8)$ \\
\hline Increased, N(\%) & $58(67.4)$ & $46(68.6)$ & $12(63.1)$ \\
\hline Reduced, N(\%) & $4(4.7)$ & $4(6)$ & $0(0)$ \\
\hline Dysplastic, N(\%) & $40(46.5)$ & $33(49.2)$ & $7(36.8)$ \\
\hline \multicolumn{4}{|l|}{ Erythroid precursors } \\
\hline Hyperplasia, N(\%) & $9(10.5)$ & $7(10.4)$ & $2(10.5)$ \\
\hline Dyserythropoiesis, N(\%) & $22(25.6)$ & 21(31.3) & $1(5.3)$ \\
\hline \multicolumn{4}{|l|}{ Lymphoid infiltrate } \\
\hline Median \% infiltrate (range) & $4(0-18)$ & $1(0-4.5)$ & $3(2-18)$ \\
\hline Absent, N(\%) & $49(57)$ & $34(50.7)$ & $15(78.9)$ \\
\hline Mixed, N(\%) & $12(14)$ & $9(13.4)$ & $3(15.8)$ \\
\hline $\mathrm{B}, \mathrm{N}(\%)$ & $3(3.5)$ & $2(3)$ & $1(5.3)$ \\
\hline T, N(\%) & 14(16.3) & $14(20.9)$ & $0(0)$ \\
\hline \multicolumn{4}{|l|}{ Fibrosis } \\
\hline MF0, N(\%) & $57(66.3)$ & $43(64.2)$ & 14(73.7) \\
\hline $\mathrm{MF} 1, \mathrm{~N}(\%)$ & 29(33.7) & $24(35.8)$ & $5(26.3)$ \\
\hline
\end{tabular}

N: number; M: male; F: female; Plt: platelet count; CR: complete response; PR: partial response; MF0: marrow fibrosis grade 0; MF1: marrow fibrosis grade 1. *Anti-platelets antibodies were tested on 54 patients, 47 on eltrombopag and 23 on romiplostim. Criteria for response were: CR, Plt $>100 \times 10^{\circ} / \mathrm{L} ; \mathrm{PR}, \mathrm{Plt}>30 \mathrm{x} 10^{9} / \mathrm{L}$. 
(ROMI) and eltrombopag (EPAG)., ${ }^{1,3}$ TPOa have shown high response rates (in up to $90 \%$ of cases) in clinical trials with comparable efficacy for both drugs. Their use in the real world showed slightly lower response rates, and interestingly, the possibility of therapy discontinuation or dose reduction in robust responders. ${ }^{3,4}$ However, little is known about predictors of response, with the exception of a greater efficacy in early phases of the disease, in nonsplenectomized patients, and in those without bleeding signs at diagnosis. ${ }^{1,5,6}$ Here we investigated the predictive value of bone marrow features on response to TPOa in a multicentric real world cohort of 86 ITP patients followed a median of 3.8 years (range: $0.4-39.6$ ). Data were retrospectively collected from clinical records and all cases were on active follow up at the time of the study. All the diagnostic procedures and investigations were performed after obtaining informed consent and approval from the Institutional Ethical Committee, and in accordance with the Declaration of Helsinki of 1975. Statistical analysis was performed with Stata15 software. Patients have been treated with EPAG $(n=67)$ and ROMI $(n=19)$ from September 2009 to May 2018, and bone marrow aspirate and biopsy have been performed before TPOa initiation.

As shown in Table 1, patients were mainly middleaged females presenting with severe thrombocytopenia, bleeding in $58.1 \%$ of cases ( $22 \%$ of grade III-IV), and positive anti-PLT autoantibodies in $52 \%$ tested cases. Baseline characteristics were comparable between the two groups (ROMI and EPAG). All cases had received first-line treatment with steroids and $43 \%$ at least a second line, including splenectomy $(23 \%)$, rituximab $(22 \%)$, cyclosporine $(19 \%)$, azathioprine $(6 \%)$, and danazol $(9 \%)$. Response rates confirm those reported in literature (steroids $68 \%$, splenectomy $70 \%$, rituximab $52 \%$, and $40-60 \%$ for the others). Bone marrow histology revealed heterogeneous cellularity, with hypocellularity in $20.9 \%$ of cases. Concerning megakaryocytes, only $67.4 \%$ displayed increased number, and about a half of cases showed dysmegakaryopoiesis. Some dysplastic features were present also in the erythroid lineage, in about one quarter of patients. As regards lymphocyte infiltrate, only $3 \%$ of cases had a detectable polyclonal B-cell population, versus about $15 \%$ with a T-cell or mixed lympho-

Table 2. New and known predictors of response to thrombopoietin receptor agonists in immune thrombocytopenia.

\begin{tabular}{lcccccc} 
Predictor & N & ROMI/EPAG & value & Study type & Follow-up on TPOa & reference \\
Early phase disease & 220 & $0 / 220$ & favorable & retrospective & 13 months (5-22) & González-López TJ $^{6}$ \\
Low TPO levels & 67 & $46 / 37$ & favorable & retrospective & 11 months (0.5-167) & Al-Samkari H $^{9}$ \\
Bleeding & 72 & $72 / 0$ & unfavorable & retrospective & 24 months (n.a.) & Khellaf M $^{1}$ \\
Splenectomy & 124 & $55 / 69$ & unfavorable & retrospective & n.a. & Mazza P $^{5}$ \\
\hline Bone marrow features* 86 & $19 / 67$ & unfavorable & retrospective & 16 months (4-130) & Present study
\end{tabular}

EPAG: eltrombopag; N: number; n.a. not available; ROMI romiplostim; TPO: thrombopoietin; TPOa: thrombopoietin analogs. *Bone marrow features correlating with lower response rates were bone marrow hypocellularity and fibrosis, megakaryocytopenia, dyserythropoiesis, presence of a T-cell infiltrate and a previous exposure to cyclosporine or azathioprine.

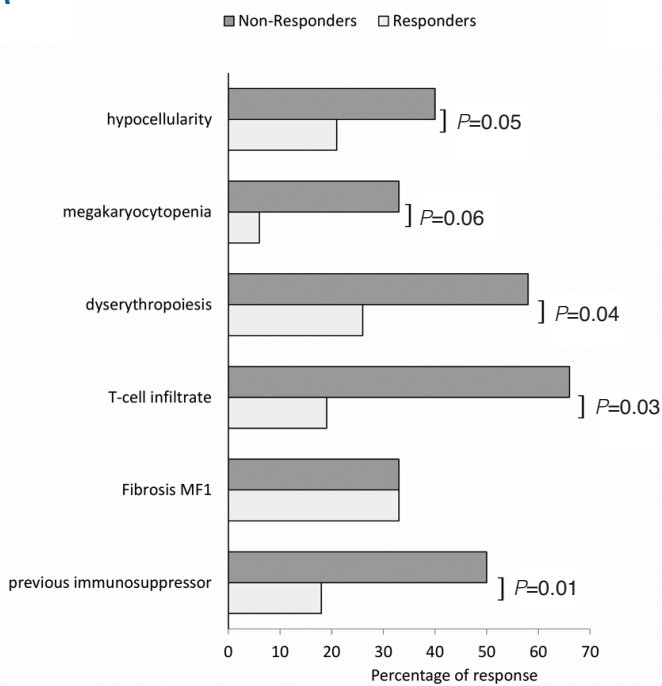

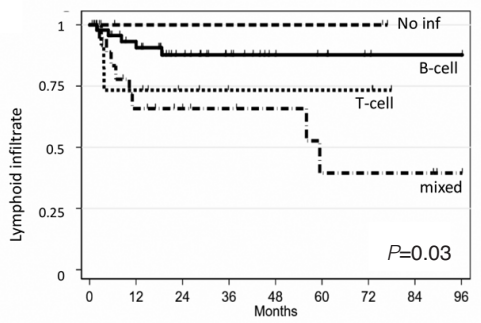

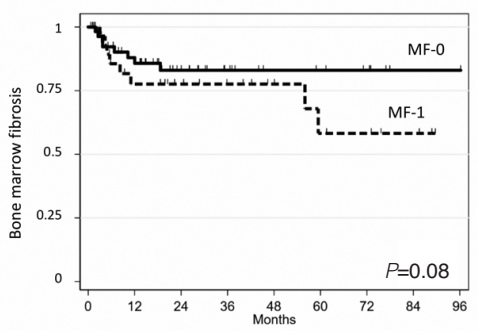

Figure 1. Predictors of response and of relapse-free survival in patients treated with thrombopoietin analogs (TPOa). (A) Percentage of responders and nonresponders according to bone marrow features or previous treatment. (B) Relapse-free survival (months) by Kaplan-Meyer method according to bone marrow megakaryocytes, lymphoid infiltrate, and reticulinic fibrosis. The following hazard ratios emerged: 2.58 (95\% Cl: $0.61-10.8)$ for T-cell infiltrate and 4.24 (95\% Cl: 1.38-13) for mixed infiltrate versus no or B-cell infiltrate, and 1.82 ( $95 \% \mathrm{Cl}$ : 0.68-4.86) for bone marrow fibrosis grade 1 (MF1). 
cyte infiltrate. Notably, one third of patients showed reticulinic fibrosis bone marrow fibrosis grade 1 (MF-1). Morphological features were comparable between groups treated with the two TPOa. Finally, all patients displayed normal karyotype, except for three cases (1 ROMI and 2 EPAG) showing a chromosome Y deletion. Median time from diagnosis to TPOa was 2.4 years (range: 0.1-28.8 years). Patients were treated for a median of 1.4 years (range: $0.3-10.8$ years), and ORR at three months and nine months were $75.6 \%$ [Complete Response (CR) 44.2 and Partial Response (PR) 31.4\%] and $65.1 \%$ (CR 36 and PR 29.1\%), respectively. Response rates to EPAG and ROMI were comparable. Regarding predictors of response, bone marrow hypocellularity, megakaryocytopenia, and dyserythropoiesis were significantly more frequent among non-responders to TPOa (Figure 1A). Other factors significantly associated with poor response were the presence of a T-cell infiltrate, or a previous exposure to cyclosporine or azathioprine. At the last follow up, 55 patients were still on treatment, whereas 31 ( $n=20$ EPAG; $n=11$ ROMI) discontinued because of lack of response or relapse $(n=17)$, adverse events or intolerance $(n=2)$; the other 12 patients, showing a robust response discontinued the drug, and seven of these are still in remission. Fourteen out of 65 responders $(21.5 \%)$, ten EPAG and four ROMI, relapsed after a median of 6.2 months (range: 1.8-60 months) and were variably managed (3 splenectomized, 1 switched from ROMI to EPAG, 1 received danazol, 5 were re-treated with EPAG, and the remaining were managed with steroids and supportive treatment). Presence of T-cell or mixed lymphoid infiltrate and reticulinic fibrosis were associated with shorter relapse-free survival (RFS) (Figure 1B). During EPAG treatment, seven adverse events occurred: two grade IV (1 stroke with platelet counts of about $30 \times 10^{9} / \mathrm{L}$, and 1 non-ST elevated myocardial infarction 1 month after EPAG discontinuation for sustained CR), one grade III pneumonia, and four grade I/II transaminase elevation. No events occurred under ROMI.

This is the first paper aimed at evaluating the predictive value of bone marrow features on the response to TPOa (Table 2). In particular, we identified hypoplasia of the megakaryocytic lineage and dysplastic features as factors associated with a poor response to TPOa. As a matter of fact, bone marrow trephine is not routinely performed in ITP. Therefore, it is not possible to exclude the possibility that a proportion of patients enrolled in the registered trials eventually belong to the idiopathic cytopenia/dysplasia of uncertain significance (ICUS/IDUS). The latter are recently described entities, characterized by borderline cytopenia/dysplasia not reaching the diagnostic threshold for myelodysplastic syndrome (MDS) and possibly preceding overt disease.' Consistently, bone marrow biopsy monitoring over time has shown a possible evolution of chronic relapsing ITP into ICUS/IDUS. ${ }^{8}$ Moreover, an additional category has been described, namely the clonal cytopenia of unknown significance (CCUS), where a marker of clonality is present. A complex genomic landscape has been reported in the ICUS/IDUS mainly involving the erythroid lineage, and a similar workup would be advisable in ITP, since the occurrence and type of somatic mutations are completely unknown. It is well established that baseline endogenous erythropoietin levels predict response to therapy with erythroid stimulating agents in MDS. In this context, it is worth mentioning a recent report showing that low TPO levels predict a greater probability and magnitude of response to TPOa. ${ }^{9}$ On the contrary, patients with extreme elevation in TPO are unlikely to respond, suggesting that baseline serum
TPO levels may be clinically useful and give insights in the variability of response to TPOa. Interestingly, EPAG was shown effective in increasing platelets and reducing bleeding in about $40 \%$ of low-risk MDS. ${ }^{10}$ Likewise, a large randomized phase II study with ROMI in low-risk MDS demonstrated reduced platelet transfusions and hematologic improvement, without increased risk of clonal evolution. ${ }^{11}$ Moreover, EPAG induced up to $40 \%$ trilineage hematologic improvement in relapsed/refractory aplastic anemia (AA), and ameliorated response to standard immunosuppression (anti-thymocyte globulin and cyclosporine), indicating its effect on the stem cell pool. ${ }^{12,13}$ On the whole, although responses are observed in MDS and AA, EPAG and ROMI are more effective in classic ITP, confirming the importance of megakaryocytic reservoir.

As far as bone marrow features are concerned, in TPOa treated cases, bone marrows were characterized by myeloproliferative neoplasm-like features, resembling those of essential thrombocythemia or occasionally early primary myelofibrosis, with increased reticulin fibrosis and trilineage hematopoiesis. ${ }^{14}$ Here, we found that reticulinic fibrosis before TPOa therapy predicted shorter RFS, underlining the detrimental role of this morphological feature. This is in line with what has already been reported in several conditions including MDS and autoimmune hemolytic anemia. ${ }^{15}$ Finally, we reported that the presence of a bone marrow $\mathrm{T}$-cell infiltrate negatively impacted on response to TPOa and RFS. At the same time, we observed a lower response rate to TPOa in patients previously treated with cytotoxic immunosuppressants (cyclosporine and azathioprine). It can be hypothesized that these cases, notwithstanding immunosuppressive therapy, still show a prominent cellular-mediated immune attack to bone marrow precursors.

In conclusion, there is growing interest in the identification of predictors of response to TPOa in several hematologic conditions. In addition to the previously reported predictors of worse outcome (late phase of the disease, previous splenectomy, bleeding signs at diagnosis, high endogenous TPO), we added bone marrow characteristics such as megakaryocytopenia, dysplastic features, reticulinic fibrosis, and $\mathrm{T}$-cell infiltrate. Therefore, bone marrow evaluation prior to initiate TPOa in ITP is advisable, as it may help in harnessing therapy and in unraveling the physiopathological mechanisms underlying TPOa refractoriness.

Bruno Fattizzo, ${ }^{1}$ Raffaella Pasquale, ${ }^{1}$ Monica Carpenedo, ${ }^{2}$ Silvia Cantoni, ${ }^{3}$ Giuseppe Auteri, ${ }^{4}$ Doriana Gramegna, Mariella D'Adda, Mariasanta Napolitano, ${ }^{5}$ Dario Consonni, ${ }^{7}$ Marco Ruggeri, ${ }^{8}$ Sergio Siragusa, ${ }^{6}$ Giuseppe Rossi, ${ }^{5}$

Nicola Vianellit and Wilma Barcellinit

'Fondazione IRCCS Ca' Granda Ospedale Maggiore Policlinico di Milano, UOC Ematologia, Milano; ${ }^{2}$ Ematologia e Trapianto, ASST Ospedale San Gerardo di Monza, Monza; ${ }^{3}$ Division of Hematology, Niguarda Cancer Center, ASST Niguarda Hospital, Milan; "Institute of Hematology "L. and A. Seràgnoli", Sant'Orsola-Malpighi University Hospital, Bologna; 'Ematologia e Dipartimento di Oncologia Clinica, A.O. Spedali Civili, Brescia; ${ }^{6}$ Università degli Studi di Palermo, Dipartimento Biomedico di Medicina Interna e Specialistica, Palermo; 'Fondazione IRCCS Ca' Granda Ospedale Maggiore Policlinico di Milano, Milano and UOC Epidemiologia, Milano and ${ }^{8}$ Dipartimento di Ematologia, Ospedale San Bortolo, Vicenza, Italy

Correspondence: WILMA BARCELLINI.

wilma.barcellini@policlinico.mi.it. doi:10.3324/haematol.2019.216804

Information on authorship, contributions, and financial \& other disclo- 
sures was provided by the authors and is available with the online version of this article at www. haematologica.org.

\section{References}

1. Khellaf M, Michel M, Quittet P, et al. Romiplostim safety and efficacy for immune thrombocytopenia in clinical practice: 2-year results of 72 adults in a romiplostim compassionate-use program. Blood. 2011;118(16):4338-4345.

2. Wang L, Li Y, Hou M. Idiopathic thrombocytopenic purpura and dysmegakaryocytopoiesis. Crit Rev Oncol Hematol. 2007;64(2):8389.

3. Rodeghiero F, Carli G. Beyond immune thrombocytopenia: the evolving role of thrombopoietin receptor agonists. Ann Hematol. 2017;96(9):1421-1434.

4. Gonzalez-Porras JR, Bastida JM. Eltrombopag in immune thrombocytopenia: efficacy review and update on drug safety. Ther Adv Drug Saf. 2018;9(6):263-285.

5. Mazza P, Minoia C, Melpignano A. The use of thrombopoietinreceptor agonists (TPO-RAs) in immune thrombocytopenia (ITP): a "real life" retrospective multicenter experience of the Rete Ematologica Pugliese (REP). Ann Hematol. 2016;95(2):239-244.

6. González-López TJ, Fernández-Fuertes F, Hernández-Rivas JA .Efficacy and safety of eltrombopag in persistent and newly diagnosed ITP in clinical practice. Int J Hematol. 2017;106(4):508-516.

7. Arber DA, Orazi A, Hasserian R, et al. The 2016 revision to the World Health Organization classification of myeloid neoplasms and acute leukemia. Blood. 2016;127(20):2391-2405.

8. Barcellini W, Fattizzo B, Zaninoni A, et al. Clinical evolution of autoimmune cytopenias to idiopathic cytopenias/dysplasias of uncertain significance (ICUS/IDUS) and bone marrow failure syndromes. Am J Hematol. 2017;92(3):E26-E29.

9. Al-Samkari H, Kuter DJ. Thrombopoietin level predicts response to treatment with eltrombopag and romiplostim in immune thrombocytopenia. Am J Hematol. 2018;93(12):1501-1508.

10. Oliva EN, Alati C, Santini V, et al. Eltrombopag versus placebo for low-risk myelodysplastic syndromes with thrombocytopenia (EQoL-MDS): phase 1 results of a single-blind, randomised, controlled, phase 2 superiority trial. Lancet Haematol. 2017;4(3):e127e136.

11. Kantarjian HM, Fenaux P, Sekeres MA, et al. Long-term follow-up for up to 5 years on the risk of leukaemic progression in thrombocytopenic patients with lower-risk myelodysplastic syndromes treated with romiplostim or placebo in a randomised double-blind trial. Lancet Haematol. 2018;5(3):e117-e126.

12. Townsley DM, Scheinberg P, Winkler T, et al. Eltrombopag Added to Standard Immunosuppression for Aplastic Anemia. N Engl J Med. 2017;376(16):1540-1550.

13. Scheinberg P. Activity of eltrombopag in severe aplastic anemia. Hematology Am Soc Hematol Educ Program. 2018;2018(1):450-456.

14. Boiocchi L, Orazi A, Ghanima W, Arabadjief M, Bussel JB, Geyer JT. Thrombopoietin receptor agonist therapy in primary immune thrombocytopenia is associated with bone marrow hypercellularity and mild reticulin fibrosis but not other stromal abnormalities. Mod Pathol. 2012;25(1):65-74.

15. Fattizzo B, Zaninoni A, Gianelli U, et al. Prognostic impact of bone marrow fibrosis and dyserythropoiesis in autoimmune hemolytic anemia. Am J Hematol. 2018;93(4):E88-E91. 